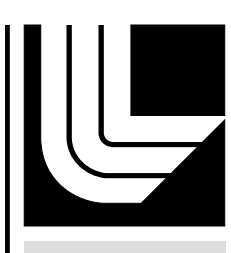

LAWRENCE LIVERM ORE NATION A L LABORATORY

Modeling Max-of-N Fluence
Distribution for Optics Lifetime

Z. M. Liao, J. Hubel, J. T. Trenholme, C. W. Carr

October 31, 2011

Proceedings of SPIE 
This document was prepared as an account of work sponsored by an agency of the United States government. Neither the United States government nor Lawrence Livermore National Security, LLC, nor any of their employees makes any warranty, expressed or implied, or assumes any legal liability or responsibility for the accuracy, completeness, or usefulness of any information, apparatus, product, or process disclosed, or represents that its use would not infringe privately owned rights. Reference herein to any specific commercial product, process, or service by trade name, trademark, manufacturer, or otherwise does not necessarily constitute or imply its endorsement, recommendation, or favoring by the United States government or Lawrence Livermore National Security, LLC. The views and opinions of authors expressed herein do not necessarily state or reflect those of the United States government or Lawrence Livermore National Security, LLC, and shall not be used for advertising or product endorsement purposes. 


\title{
Modeling max-of-n fluence distribution for optics lifetime
}

\author{
Zhi M. Liao, John Hubel, John B. Trenholme, C. Wren Carr \\ Lawrence Livermore National Laboratory, 7000 East Avenue, P. O. Box 808, \\ Livermore, CA, USA 94551
}

\begin{abstract}
Local temporal shot-to-shot variation of a high-energy laser system is measured in order to model the maximum fluence that any location on the optic will be exposed to after $\mathrm{N}$ shots (Max-of-N). We constructed a model to derive an equivalent-Max-of-N fluence distribution from a series of shots of differing energy and contrast in order to calculate damage initiation and optics lifetime. This model allows prediction for Max-of-N effects when direct measurements of the fluence distribution are not available. Comparison to different laser systems will be presented in order to gain insight as to the physical origins of the Max-of-N effect.
\end{abstract}

Keywords: Laser-induced optic damage, optics lifetime, laser contrast, statistical optics, laser beam characterization

\section{INTRODUCTION}

The National Ignition Facility (NIF) is the most energetic pulsed laser system in the world with $1.8 \mathrm{MJ}$ of energy at $355 \mathrm{~nm}$ or $3 \omega$. NIF uses laser-damage-resistant optics to withstand the high laser fluence ${ }^{1}$. NIF has developed optics lifetime models to predict when an optic needs to be refurnished or replaced as part of an integrated operational strategy. Optic lifetime depends on the number of initiation sites and their rate of growth. The first step in modeling an optic's lifetime is calculating the number of initiations it accumulates over a sequence of shots. The number of initiations can be calculated given the laser fluence probability density function (pdf), $f(\phi)$ and the fluence-dependent damage density ${ }^{2}$, $\rho(\phi)$ as follows

$$
N_{\text {init }}=A \cdot \int \rho(\phi) \cdot f(\phi) \cdot d \phi,
$$

with A being the area of the optic. This is straight forward for a single shot but when the optic is shot many times, the fluence distribution has to account for the accumulated effects of shot-to-shot contrast. This is what we describe as the Max-of-N effect and it is crucial in the modeling of optics lifetime because shot-to-shot dynamics can substantially increase the number of initiations and therefore the optics lifetime. For example, Figure 1 shows an example of a series of repeated shots. Given the fluence pdf of the first shot and the optic's response, the number of initiations can be calculated using Eq. 1. Since subsequent shots have the same energy and contrast, fixed-beam-profile calculations would yield no additional initiations since the first shot has created all the initiations and subsequent shots are not hotter at any location. However, the energy and contrast don't account for the "movement of hot spots" from shot to shot and the resultant shift of the accumulated maximum (i.e. Max-of-N) fluence as shown in Figure 1. This results in a higher number of initiations as shots are increased. The Max-of- $\mathrm{N}$ fluence is the maximum fluence at a given location over the course of $\mathrm{N}$ shots. This can be defined mathematically as

$$
\phi_{\text {MaxN }}(x, y)=\underset{i=1 . . N}{\operatorname{Max}}\left\{\phi_{i}(x, y)\right\},
$$

with $\phi_{\text {MaxN }}$ being the Max-of- $\mathrm{N}$ fluence, $\phi_{\mathrm{i}}$ is the measured fluence, $\mathrm{i}$ is the shot index, and $\mathrm{N}$ is the total number of shots. Max-of-N comes from the shot-to-shot (dynamic) random fluctuation of local laser fluence. Without the dynamic contrast, the Max-of-N fluence would be identical to the individual measured fluence. With Max-of-N, a sequence of shot fluences can be replaced with a single fluence in calculating the number of initiations under the following condition:

1. Damage precursors are stationary (i.e. they do not move from shot to shot) with respect to the beam. 
2. Damage behavior is deterministic (i.e. the precursors will be damaged the first time it is expose to a fluence greater than the damage threshold fluence in all instances).

3. Damage precursors do not exhibit laser conditioning (i.e. they do not change their damage behavior with prior laser fluence) such as observed in fused silica ${ }^{3}$ but not seen in (e.g.) KDP.

4. Damage precursors do not exhibit damage fatigue (i.e. the damage threshold does not decrease with increasing number of shots).
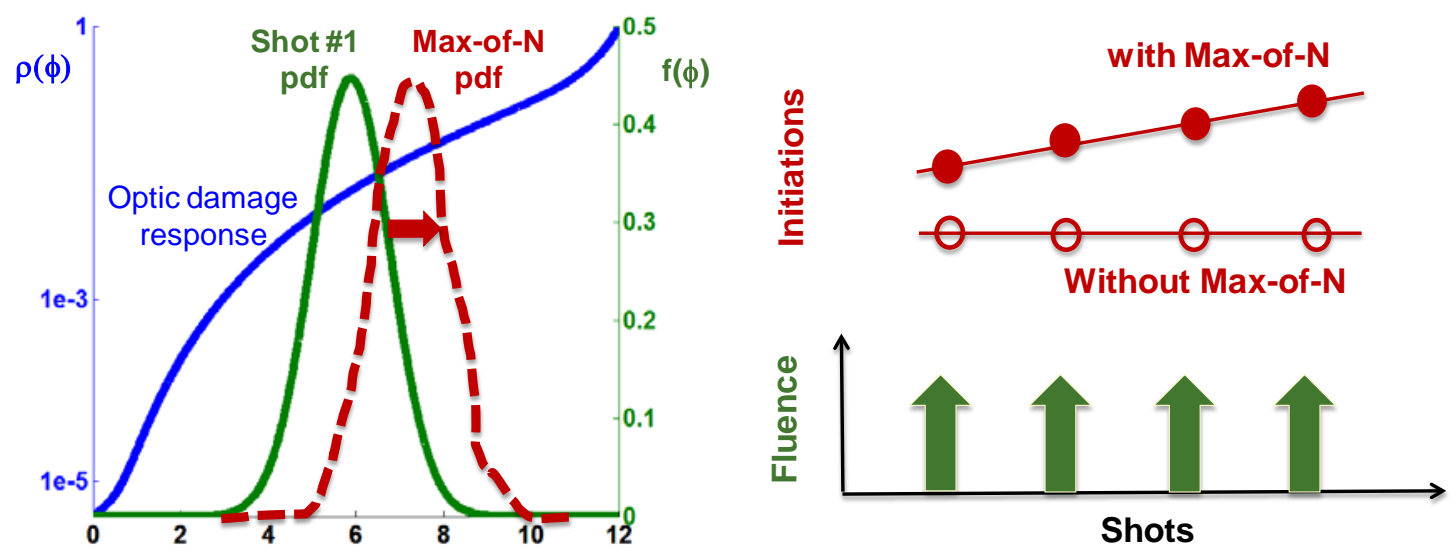

Figure 1. Plot of optic damage response $\rho(\phi)$ (line) and the single shot (line) and Max-of-N (dash) pdf (left) and schematic showing effect of Max-of-N on number of initiations on a series of repeated shots (right).

\section{METHODOLGY}

We have taken measurements from a large-aperture test beam $\left(\sim 1000 \mathrm{~cm}^{2}\right)$ for detailed analysis of the evolution of fluence distributions. The test beam has both a $1 \omega$ near field (NF) and a $3 \omega$ NF profile (fluence map) that comes from frequency converting the $1 \omega$ beam. Consecutive shot sequences, where laser operations were fixed (i.e. pulse shape, energy, beam size, etc.), were analyzed for shot-to-shot dynamics. Several data sets (see Table 1) were analyzed in an attempt to capture the dependency of the Max-of- $\mathrm{N}$ effect on different operation regimes. We model Max-of- $\mathrm{N}$ fluence distribution using the measured value of shot-to-shot contrast ${ }^{4}$. This model assumes that the Max-of-N fluence distribution of a series of $\mathrm{N}$ consecutive repeated shots is just the single shot fluence distribution $\mathrm{f}_{\mathrm{S}}$ (either Gaussian or Rician) with a modified mean $\mu_{\max }$ and same spatial fluence standard deviation. The Max-of $\mathrm{N}$ fluence distribution can be modeled by a Gaussian or Rician probability function

$$
f_{\max }(\phi)=f_{S}\left(\phi ; \mu_{\max }\left(N, \mu, \sigma_{T}\right), \sigma_{S}\right)
$$

with $\phi$ being the local fluence, $\mu$ being the fluence mean, $\sigma_{\mathrm{S}}$ being the spatial fluence standard deviation, and $\sigma_{\mathrm{T}}$ being standard deviation of the shot-to-shot fluctuation. The single shot fluence distribution $\mathrm{f}_{\mathrm{S}}$ in Gaussian form is

$$
f_{S}\left(\phi ; \mu, \sigma_{S}\right)=\frac{1}{\sigma_{S} \sqrt{2 \pi}} e^{-\frac{(\phi-\mu)^{2}}{2 \sigma_{S}^{2}}} \text {. }
$$

The Max-of-N mean fluence $\mu_{\max }$ is the mean fluence of the probability density function of the maximum in a series of $\mathrm{N}$ shots, $\mathrm{f}_{\mathrm{N}}$ given $\mathrm{by}^{5}$ 


$$
f_{N}\left(x ; \phi, \sigma_{T}, N\right)=N \cdot f_{T}\left(x ; \phi, \sigma_{T}\right) \cdot F_{T}\left(x ; \phi, \sigma_{T}\right)^{N-1}
$$

with the shot-to-shot fluence probability density function $\mathrm{f}_{\mathrm{T}}$ and associated cumulative density function $\mathrm{F}_{\mathrm{T}}$. The shot-toshot fluence distribution can be modeled by a Gaussian (or Rician) probability function with different mean and standard deviation than Eq. 4,

$$
f_{T}\left(x ; \phi, \sigma_{T}\right)=\frac{1}{\sigma_{T} \sqrt{2 \pi}} e^{-\frac{(x-\phi)^{2}}{2 \sigma_{T}^{2}}} .
$$

This simple model is quite powerful in its functionality. It can model the fluence pdf over a series of arbitrary shots with only 1 parameter $\left(\sigma_{\mathrm{T}}\right)$ that is not easily measured, the other inputs (fluence mean, spatial contrast) are readily available or straight-forward to measure in most systems. For optics lifetime predictions, the number of initiations an optic accumulated its lifetime can be calculated as in Eq.1 but simply replacing the single-shot fluence pdf (f) by the Max-of$\mathrm{N}$ fluence pdf $\mathrm{f}_{\max }$ as given in Eq. 3.

\section{EXPERIMENT DATA \& RESULTS}

\subsection{Spatial fluence distribution}

An example of a shaped long-pulse, 3w near field patch obtained at high power is displayed in Figure 2 along with measured fluence pdf. The prominent structures in the displayed fluence map are due to the presence of a continuous phase plate installed in front of the frequency converter at the time. This fluence pdf is also plotted against a Gaussian model with a mean of $5.8 \mathrm{~J} / \mathrm{cm}^{2}$ and standard deviation of $0.9 \mathrm{~J} / \mathrm{cm}^{2}$. It is evident from the figure that a Gaussian model is a good fit for the fluence pdf.
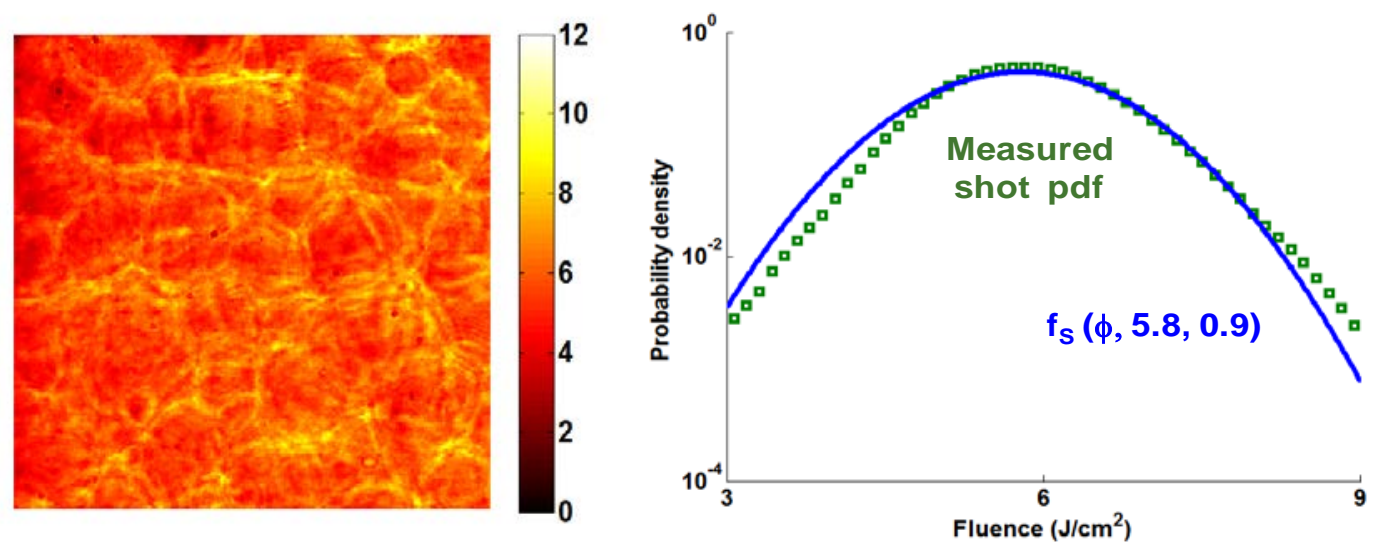

Figure 2. Large-aperture 3w fluence near field fluence map is plotted on left. The empirically derived fluence pdf (square) and its associated Gaussian pdf model (line) with a mean of $5.8 \mathrm{~J} / \mathrm{cm}^{2}$ and standard deviation of 0.9 $\mathrm{J} / \mathrm{cm}^{2}$ is plotted on the right.

\subsection{Shot-to-shot fluence distribution}

The Max-of-N fluence effect is a product of the shot-to-shot fluence fluctuation. To measure the shot-to-shot fluence distribution, we analyze the fluence changes at each location of the fluence near field (see Figure 3) with a spatial resolution of $\sim 0.2 \mathrm{~mm}^{2}$. We find that the fluence fluctuation is nearly constant across the beam and that value quickly converges after only a few shots. The value of shot-to-shot contrast (i.e. shot-to-shot standard deviation divided by the 
mean fluence) for the different shot sequences analyzed is listed in Table 1. For the series of shaped long-pulse $3 \omega$ shots, the shot-to-shot standard deviation is approximately $8.5 \%$ of the mean fluence of the shot. This value varies with different laser operation condition (see Table 1). We believe that the higher value of shot-to-shot contrast for the $3 \mathrm{w}$ beam as compared to the fundamental $1 \mathrm{w}$ beam is the added contrast from the nonlinear frequency conversion process. Furthermore, the slightly higher shot-to-shot contrast of the Flat-In-Time (FIT) short-pulse sequence as compared to the shaped long-pulse sequence comes from the fact that the laser amplifiers were deep in saturation during long-pulse operation, and this reduced contrast.
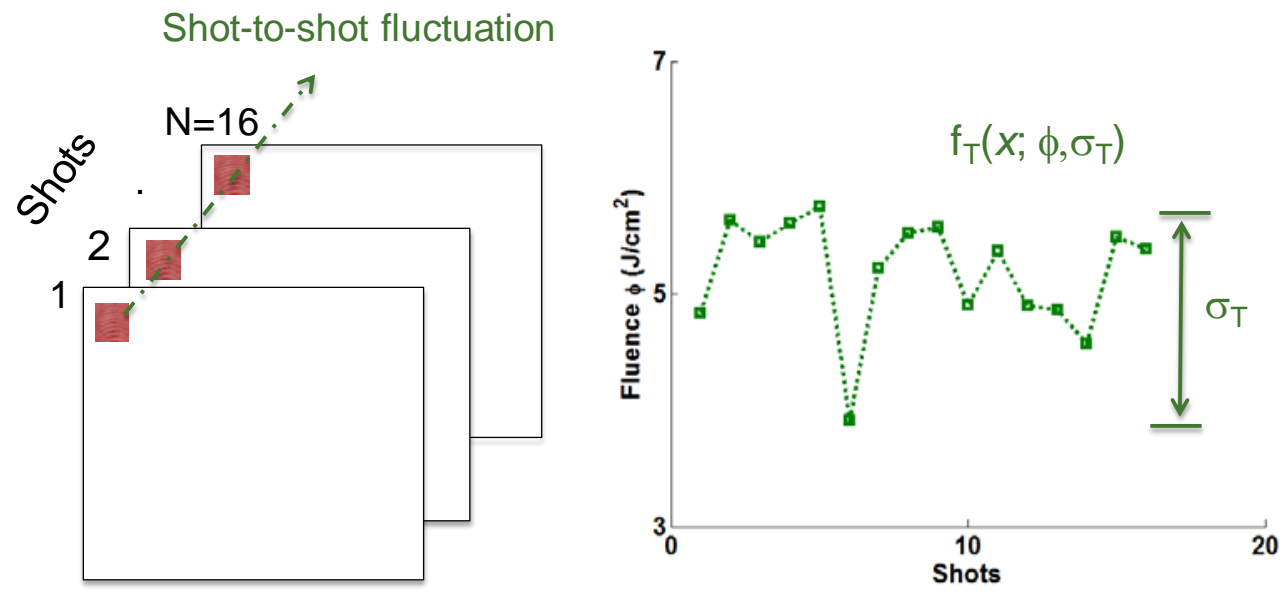

Figure 3. Schematic of the shot-to-shot fluctuation measurement (left). Example of the shot-to-shot fluence fluctuation at a given near field location over 16 shots (right).

Table 1. Shot sequences analysis for Max-of-N effect.

\begin{tabular}{|c|c|c|c|}
\hline Sequence & Number of Shots & Frequency & Shot-to-Shot Contrast \\
\hline Shaped Long-Pulse (15ns) & 16 & $1 \omega$ & $3.4 \%$ \\
\hline Shaped Long-Pulse (15ns) & 16 & $3 \omega$ & $8.5 \%$ \\
\hline FIT Short-Pulse (1ns) & 12 & $1 \omega$ & $4.2 \%$ \\
\hline FIT Short-Pulse (1ns) & 12 & $3 \omega$ & $9.0 \%$ \\
\hline
\end{tabular}

The 3w near-fields of each of the 16 shots were analyzed using a Gaussian pdf model. The mean and standard deviation as a function of shot number were found. As each shot is added to the sequence, the accumulated Max-of-N $3 \mathrm{w}$ nearfield is calculated (see Eq. 2) for all spots in the beam and its Max-of-N mean is plotted for comparison (see Figure 4). It is evident from the plot that although the measured mean of each shot is constant with respect to shot number, the Max-of-N mean fluence increases with shot number. This is the Max-of-N effect and it comes from the dynamic shotto-shot contrast of the laser. Our model was able to reproduce the result of the increasing mean fluence as well as the shape of the probability density function (see Figure 4). 

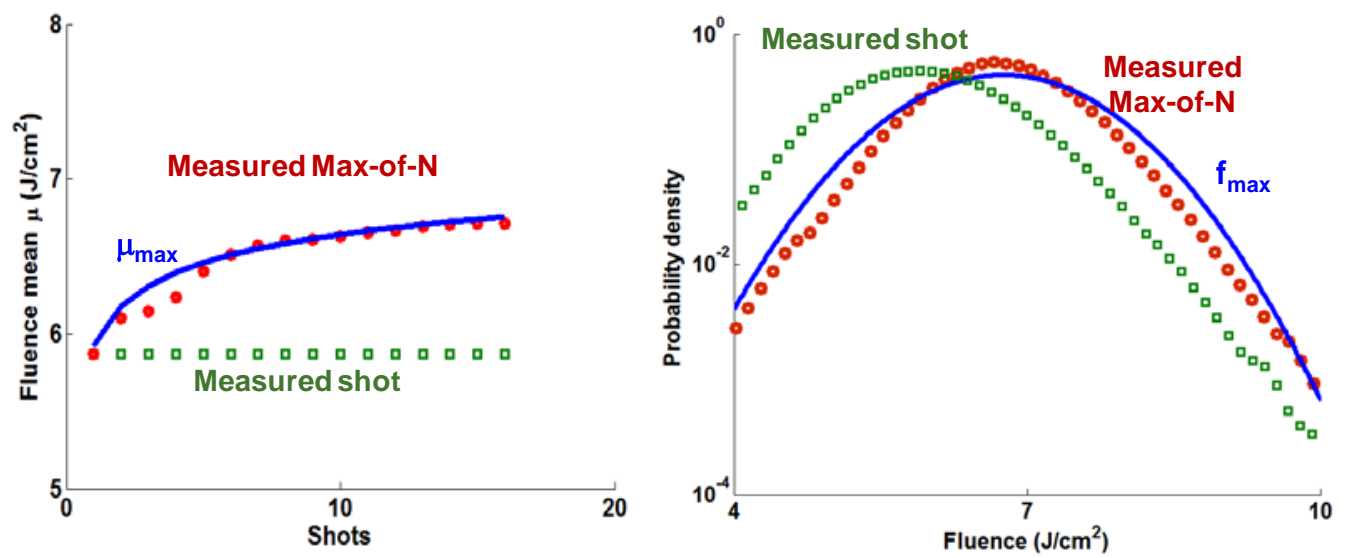

Figure 4. Mean fluence of each measured shot (square), measured Max-of-N shot (point), and the model prediction $\mu_{\max }$ (line) are plotted as a function of shots on the left. Probability density of measured shot (square), Max-of-N shot (for 16 shots) (point), and the model $\mathrm{f}_{\max }$ (line) are plotted vs. fluence on the right.

\subsection{Arbitrary pulse sequence for a large-aperture laser system}

In order for the model to work with an arbitrary series of pulses with different energy and pulse shapes, we have developed an algorithm called EquiviMax that uses our Max-of-N model to return a Max-of-N fluence distribution for any arbitrary series of shots. EquiviMax accomplishes this task by taking an arbitrary series of $\mathrm{N}$ shots and

1. Scaling the fluence by appropriate scaling factors to account for pulse shape dependence ${ }^{6}$

2. Sorting the shot sequence into an ascending order

3. Segmenting the shot series into groups of nearly identical fluence shots

4. For each group/segment of identical shots, applying Max-of-N model to return a segment Max-of-N fluence

5. Finding the largest segment Max-of-N fluence, this is the EquiviMax fluence

A series of 28 shots of varying energy with 4 different pulse shapes from the large-aperture test beam $\left(\sim 1000 \mathrm{~cm}^{2}\right)$ was used to test EquiviMax (see Figure 5). EquiviMax divided the shot series into 4 segments and the calculated Max-of-N mean fluence is plotted vs. the initial unsorted shot series (Figure 5). This shot sequence was also the entire shot history of a test optic; as a result, we can calculate the number of initiations the part accumulates using Eq 1 . by replacing fluence pdf by Max-of-N fluence pdf with mean fluence as calculated by EquiviMax. The number of initiations as calculated using EquiviMax result is 9 which is in excellent agreement since the optic was found to have 8 initiations after scanning with microscope. In contrast, without accounting for Max-of-N effect, the lifetime initiation of the test optic could have been estimated using only the hottest shot of the sequence which would be shot \#26 with a mean fluence only $6.5 \mathrm{~J} / \mathrm{cm}^{2}$. This calculation would result in only 3.5 initiations, which would underestimate the number of initiations by a factor of two. This discrepancy could be even more as the Max-of-N fluence of this optic was driven by the hottest shot sequence which only had 3 shots (the last three shots in the series), therefore the Max-of-N fluence was still relatively close to any of the 3 hottest shots. If the optic were to be exposed to tens of shots in the hottest sequence, the number of initiations calculated using Max-of- $\mathrm{N}$ as compare to the hottest shot would be even more dramatic.

\subsection{Arbitrary pulse sequence for a small-aperture laser system}

We also applied our EquiviMax algorithm to a series of shots produced by a small-aperture laser system. This laser system is a similar Nd:glass solid-state laser but it has a circular beam geometry and a much smaller beam size $(\sim 1$ $\mathrm{cm}^{2}$ ). This laser does not have the stability of the large-aperture test beam so we did not have consecutive repeated shot series to measure the shot-to-shot contrast. We use a shot-to-shot contrast of $10 \%$ in our EquiviMax algorithm which produced the result that is consistent with the measured Max-of-N fluence (see Figure 5). 

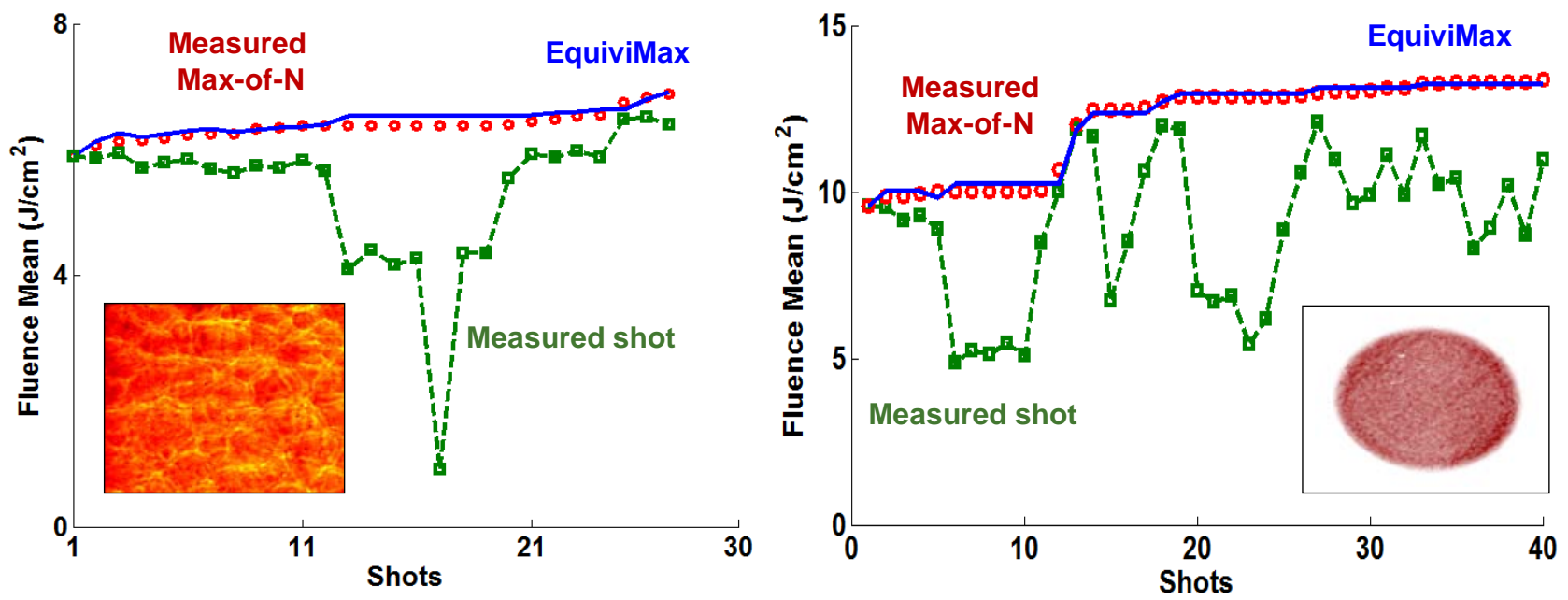

Figure 5. Analysis for shots with varying fluence for large-aperture laser with mean fluence of each measured shot (square-dash), measured Max-of-N shot (point), and the model prediction $\mu_{\max }$ (line) are plotted as a function of shots on left. Analysis for shots with varying fluence for small-aperture laser with mean fluence of each measured shot (square-dash), measured Max-of-N shot (point), and the model prediction $\mu_{\max }$ (line) are plotted as a function of shots on right. The insert display the geometry of each beam profile (not to scale).

\section{CONCLUSION}

We have successfully developed a Max-of-N model that deals with the shot-to-shot fluence fluctuations in laser systems. Our measurement of a large-aperture test beam indicated that the shot-to-shot contrast is $\sim 9 \%$ in the converted third harmonic wavelength ( $\sim 4 \%$ in the fundamental wavelength). We have developed and verified an algorithm called EquiviMax that can produce the Max-of-N fluence of an arbitrary series of shots of a laser system. We believe that this methodology and model is applicable to any laser system in general and provides a crucial capability in dealing with optics lifetime prediction.

\section{REFERENCES}

[1] Suratwala, T. I., Miller, P. E., Bude, J. D., Steele, W. A., Shen, N., Monticelli, M. V., Feit, M. D., Laurence, T. A., Norton, M. A., Carr, C. W., and Wong, L. L., "HF-based etching processes for improving laser damage resistance of fused silica optical surfaces,” J. Am. Ceram. Soc., 94, 416-428 (2011).

[2] Carr, C. W., Feit, M. D., Nostrand, M. C., and Adams, J. J., "Techniques for qualitative and quantitative measurement of aspects of laser-induced damage important for laser beam propagation,” Meas. Sci. Technol. 17, 1958 (2006).

[3] Cross, D., Carr, C. W., private communication (2011).

[4] Liao, Z. M., Huebel, J., Trenholme, J. T., Carr, C. W., "Modeling max-of-N fluence distribution using measured shot-to-shot beam contrast," Applied Optics 50, 3547-3552 (2011).

[5] Gumbel, E. J., "Extreme Value Theory”, Columbia University Press, (1958).

[6] Carr, C. W., Trenholme, J. B., Spaeth, M. L., "Effect of temporal pulse shape on optical damage,” Appl. Phy. Lett., 90, 041110 (2007).

\section{ACKNOWLEDGEMENT}

This work was performed under the auspices of the U. S. Department of Energy by Lawrence Livermore National Laboratory under Contract DE-AC52-07NA27344. (LLNL-JRNL-509595) 